\title{
Personal time management: a suggested approach for trainees in psychiatry
}

\author{
Christine Johnson, Lecturer in Mental Health, University of Bristol, 41 St Michaels \\ Hill, Clifton, Bristol BS2 8DZ
}

The concept of personal time management does not, perhaps, generate immediate enthusiasm among junior doctors: the terminology seems somewhat alien and the idea more relevant to the world of business than that of clinical practice. There is a lack of coverage of this issue in the citations concerning the applications of time management to the medical profession. Although allied professions have made an attempt to address the matter (Shubin, 1988), they have tended to concentrate more on personality types (McAlvanah, 1989) and obstacles to effectiveness (Sheridan, 1988), rather than providing practical guidelines.

There are, however, clear reasons why a time management schedule can be useful and to a trainee's advantage. As we are all aware, increasing emphasis is being placed on accountability, which includes the effective use of time. By dividing the timetable into a number of general elements, it becomes easier to audit and to make meaningful comparisons with other posts. In addition, a time management schedule can improve the quality of training.

Rotational schemes in psychiatry operate on the broad principle that suitable experience is best gained by combining supervised clinical activities with an organised postgraduate teaching programme. While this may hold true in general, in practice there is a great variation between posts in terms of, for example, the size and type of the caseload and the amount of structure to a job; few are tailored to individual priorities. Valuable time can be lost in orientating oneself to a new post and essentially filling the gap left by the previous trainee. But, by structuring the time available many of these difficulties can be avoided. Trainees could learn about the requirements of the post from previous trainees' management sheets and through discussion at scheduled team meetings. Itemising the various elements contained within a particular post will facilitate a review of the balance between service commitments and training experience, and any adjustments to the timetable can be made as necessary. The new junior doctor would then have a chance to include his or her personal priorities in terms of training within the existing framework.

What follows is a suggested approach to personal time management, which I hope is easy to use

and might at least allow trainees to gain an overview of the jobs they are actually doing if nothing else.

\section{The time management schedule}

(a) The aim. This is to establish the most efficient balance between service commitment and training needs within a defined time framework.

(b) Framework. Most trainees are employed on a full-time basis and therefore the framework would be one of ten sessions: for those working part-time it would be five sessions.

(c) First objectives. Within any clinical post, there are a number of fixed, non-negotiable elements which include ward rounds, out-patient clinics, consultant supervision and attendance at training and audit meetings.

(d) Second objective. This would be to determine individual aims for the attachment period, and can be divided into general and specific. General aims should cover the area of clinical experience to be gained in the post, and it is suggested that a basic check-list be compiled during a consultant supervision session. Specific aims will relate to the particular stage of training and might include MRCPsych examinations, psychotherapy and research. It is suggested that these are ranked in order of priority, and that at least the first priority is incorporated into the schedule as a fixed, non-negotiable element.

(e) Third objective. The remaining sessions can be seen as negotiable elements which may be divided into relatively fixed elements and floating elements. Relative fixed elements cover aspects of work that will need to be done each week, but not necessarily at a fixed time. Examples of these include seeing new admissions (average number per week to be calculated, and amount of time required), administration (at least one half session per week), and ward work (including 'therapy' sessions and keyworker role).

Floating elements refer to the remaining session time which is not allocated to particular activities, but that can be used to take advantage of training opportunities and to ensure good patient care. 


\section{Comments}

(a) It is essential that all elements (fixed, relatively fixed and floating) are represented within the schedule. If this is not possible, the suitability of the post is questionable. Indications for the size of the caseload would follow from the time available, the range of experience required and the tasks expected of the junior doctor in post.

(b) Devising the schedule with the supervising consultant should take place within the first week of starting, if not before, and should be determined by the previous trainee's experience and the individual's own training needs.

(c) The format would enable training posts to be reviewed and audited. Each trainee could monitor the schedule on a weekly basis and plan the forthcoming week, while consultants might assess it every three months. Clinical tutor reviews should take place within the attachment period on at least one occasion.

\section{Conclusion}

The potential importance of personal time management has been presented, as has a suggested practical approach towards implementing a schedule. The aim of this approach is to balance individual training needs with service commitments in a way that is open to review.

\section{References}

McAlvanah, M. F. (1989) Time management: a key to fulfilling job expectations. Paediatric Nursing, 14, 536.

SHERIDAN, M. S. (1988) Time management in health care social work. Social Work in Health Care, 13, 91-99.

ShuBIN, S. (1988) Making the most of your time. Nursing Life, 8, 38-41.

\section{MERCK ESSAY PRIZE}

Two runner up prizes of $\$ 100$ each. All entrants will receive a book token.

All trainee psychlatrists (Senior House Officers, Registrars or Senior Registrars) in the United KIngdom and Republic of Ireland are Invited to submit a 2000-3000 word essay on the topic:

\section{"Depression: Counting the Costs"}

Closing date for entries 31st March 1993

Sponsored by E. Merck Pharmaceuticals. A division of Merck Ltd. 